\title{
The dimensionality of individual niche variation
}

\author{
Travis Ingram, ${ }^{1,4}$ Raul Costa-Pereira, ${ }^{2,3}$ and Márcio S. Araújo ${ }^{3}$ \\ ${ }^{1}$ Department of Zoology, University of Otago, 340 Great King Street, Dunedin 9016 New Zealand \\ ${ }^{2}$ Programa de Pós-graduação em Ecologia e Biodiversidade, Instituto de Biociências, \\ Universidade Estadual Paulista (UNESP), Av. 24-A, 1515, Rio Claro 15807 Brazil \\ ${ }^{3}$ Instituto de Biociências, Universidade Estadual Paulista (UNESP), Av. 24-A, 1515, Rio Claro 15807 Brazil
}

\begin{abstract}
The inherently multidimensional nature of the niche has not yet been integrated into the investigation of individual niche specialization within populations. We propose a framework for modeling the between- and within-individual components of the population niche as a set of variance-covariance matrices, which can be visualized with ellipses or ellipsoids. These niche components can be inferred using multiple response mixed models, and can incorporate diverse types of data, including diet composition, stable isotopes, spatial location, and other continuous measures of niche dimensions. We outline how considering both individual and population niches in multiple dimensions may enhance our understanding of key concepts in ecology and evolution. Considering multiple dimensions as well as the within-individual component of variation can lead to more meaningful measures of niche overlap between species. The impact of a population on its food web or ecosystem can depend on the degree of individual variation (via Jensen's inequality), and we suggest how the dimensionality of individual specialization could amplify this effect. Finally, we draw from concepts in quantitative genetics and the study of animal personalities to propose new hypotheses about the ecological and evolutionary basis of niche shifts in multiple dimensions. We illustrate key ideas using empirical data from sea otters, wetland frogs, and threespine stickleback, and discuss outstanding questions about the consequences of multidimensional niche variation. Setting variation among individuals in an explicitly multivariate framework has the potential to transform our understanding of a range of ecological and evolutionary processes.
\end{abstract}

Key words: individual specialization; Jensen's inequality; multidimensional niche; niche overlap; niche shift; quantitative genetics; stable isotope analysis.

\section{INTRODUCTION}

The ecological niche of an organism defines how it interacts with all aspects of its environment, and is thus an inherently multidimensional construct (Hutchinson 1957). Niches have traditionally been modeled as properties of populations or species, but it is increasingly apparent that many populations are collections of ecologically heterogeneous individuals that vary in how they interact with their environment (Bolnick et al. 2003, 2011, Araújo et al. 2011). Individual specialization is a form of intraspecific niche variation that occurs when individuals only use a subset of the population's resource base for reasons not attributable to sex, age class or discrete morph. The realization that individual specialization is

Manuscript received 5 April 2017; revised 26 September 2017; accepted 7 December 2017. Corresponding Editor: Sebastian J. Schreiber.

${ }^{4}$ E-mail: travis.ingram@otago.ac.nz widespread necessitates a re-think of niche concepts to ensure that intraspecific variation is adequately modeled. In particular, the study of individual specialization has not been fully integrated with the concept of the niche as a multidimensional ecological space.

A multidimensional view of individual specialization has the potential to transform our understanding of niche dynamics and to bring together disparate approaches including stable isotope analysis, spatial ecology, quantitative genetics, and trait-based community ecology. The development of new technologies and methods has enhanced our ability to measure the use of resources and space by individuals at increasingly fine scales. For example, refinements to the use of ecological tracers such as stable isotopes allow the estimation of trophic niche parameters at multiple time scales (Layman et al. 2012). At the same time, tracking technology and remote sensing techniques allow measurements of the behavior and space use of individuals with ever-greater precision and for longer periods (Pimm 
et al. 2015). Many recent studies using these and other methods have produced data for numerous species encompassing multiple dietary, habitat or behavioral dimensions (Fig. 1). With an appropriate and explicitly multivariate conceptual and analytic framework, these data sources can be used to ask fundamental questions about the dimensionality of individual variation.

In this article, we outline a conceptual framework for investigating individual specialization in multiple dimensions, and explain how this perspective adds to our understanding of ecological and evolutionary processes. We develop two related arguments: that robust measures of individual niche variation should consider multiple niche axes and their covariances rather than each axis in isolation, and that studies of multidimensional resource use should integrate both the between- and within-individual components of niche variation. We show how this framework can alter predictions about key outcomes including the extent of niche overlap between species (particularly in stable isotope studies), the effect of individual variation on population mean processes and interactions (e.g., via Jensen's inequality), and the expected trajectory of niche shifts under ecological change. Along the way, we illustrate these ideas using analyses of empirical and simulated niche data, and propose several novel and testable hypotheses about the consequences of multidimensional individual specialization.
A Framework for InVestigating Multidimensional INDIVIDUAL SPECIALIZATION

In what follows, we define the niche in terms of resource use: discrete resource types, or more often continuous measures such as prey size or water depth. This usage aligns with the "classical niche theory" that is the basis of numerous ideas pertaining to niche width, niche overlap, community assembly and speciation (MacArthur and Levins 1967, Pianka 1973, Whittaker et al. 1973, Dieckmann and Doebeli 1999, Ackerly and Cornwell 2007). We acknowledge the value of other conceptions of the niche such as species' requirements for and impacts on discrete 'niche factors' (Tilman 1982, Chase and Leibold 2003, Letten et al. 2016), and see value in the exploration of how multidimensional individual specialization affects other elements of niche theory.

Individual specialization in resource use can be measured by partitioning a population's total niche width (TNW) into two additive components: the within-individual component (WIC) and the between-individual component (BIC). The WIC is the average breadth of resources used by each individual in the population, while the BIC is the variation among individuals in average resource use (Fig. 2A). Either narrower (low WIC) or more segregated (high BIC) realized niches of individuals can therefore increase the degree of individual

\begin{tabular}{|c|c|c|c|}
\hline Diet and habitat & $\begin{array}{l}\text { Multiple habitat } \\
\text { dimensions }\end{array}$ & & ted \\
\hline $\begin{array}{l}\text { DIET AND MICROHABITAT } \\
\text {-European eel (Cucherousset et al. 2011) } \\
\text {-Threespine stickleback (Snowberg et al. 2015) } \\
\text { DIET AND INSHORE VS. OFFSHORE HABITAT } \\
\text {-Arctic charr (Knudsen et al. 2010; Hawley et al. 2016) } \\
\text {-Australian sea lion (Lowther \& Goldsworthy 2011) } \\
\text {-Brown trout (Brodersen et al. 2012) } \\
\text {-Eurasian perch (Quevedo et al. 2009; Svanbäck et al. 2015) } \\
\text {-Southern sea lion (Baylis et al. 2015) } \\
\text { DIET AND FORAGING ROUTE/AREA } \\
\text {-Antarctic fur seal (Kernaléguen et al. 2012) } \\
\text {-Australian fur seal (Kernaléguen et al. 2015) } \\
\text {-Desertas petrel (Ramirez et al. 2016) } \\
\text {-Loggerhead sea turtle (Hatase et al. 2010; Peckham et al. 2011) } \\
\text { - Northern gannets (Wakefield et al. 2015) } \\
\text {-Southern elephant seal (Mclntyre et al. 2010; Martin et al. 2011) } \\
\text {-Subantarctic fur seal (Kernaléguen et al. 2012) } \\
\text {-Wandering albatross (Ceia et al. 2012) } \\
\text { DIET AND MACROHABITAT/ECOSYSTEM TYPE } \\
\text {-American alligator (Rosenblat \& Heithaus 2011) } \\
\text {-Antarctic fur seal (Casper et al. 2010) } \\
\text {-Bottlenose dolphin (Rossman et al. 2015) } \\
\text {-Dolphin gull (Masello et al. 2013) } \\
\text {-Gray snapper (Hammerschlag-Peyer \& Layman 2010) } \\
\text {-Great egret (Voslamber et al. 2010) } \\
\text {-Schoolmaster (Hammerschlag-Peyer \& Layman 2010) } \\
\text {-Sea otter (Newsome et al. 2015) } \\
\text {-White fish (Harrod et al. 2010) }\end{array}$ & $\begin{array}{l}\text {-New Zealand sea lion (Chilvers } \\
\text { 2008; Chilvers \& Wilkinson 2009) } \\
\text { Southern elephant seal (Mclntyre } \\
\text { et al. 2010) } \\
\text {-Threespine stickleback } \\
\text { (Snowberg et al. 2015) }\end{array}$ & $\begin{array}{l}\text { MULTIPLE STABLE } \\
\text { ISOTOPE DIMENSIONS AND } \\
\text { PREY SIZE } \\
\text {-Sea otter (Newsome et al. } \\
\text { 2009) } \\
\text {-Thin-toed frogs (this study) }\end{array}$ & $\begin{array}{l}\text { FORAGING BEHAVIOR AND PREY } \\
\text { TYPE } \\
\text {-Antarctic fur seal (Casper et al. 2010) } \\
\text {-Australian fur seal (Kernaléguen et } \\
\text { al. 2015) } \\
\text {-Australian sea lion (Lowther \& } \\
\text { Goldsworthy 2011) } \\
\text {-Desertas petrel (Ramirez et al. 2016) } \\
\text {-Great cormorant (Potier et al. 2015) } \\
\text {-Guillemots (Woo et al. 2008) } \\
\text {-Loggerhead sea turtle (Hatase et al. } \\
\text { 2007) } \\
\text {-New Zealand sea lion (Chilvers 2008; } \\
\text { Chilvers \& Wilkinson 2009) } \\
\text {-Northern gannets (Wakefield et al. } \\
\text { 2015) } \\
\text {-Southern elephant seal (Mclntyre et } \\
\text { al. 2010; Martin et al. 2011) } \\
\text {-Southern sea lion (Baylis et al. 2015) } \\
\text {-Wandering albatross } \\
\text { (Ceia et al. 2012) }\end{array}$ \\
\hline
\end{tabular}

FIG. 1. Examples of vertebrate populations in which niche variation is known or likely to occur in multiple ecological dimensions, and which could be studied in an explicitly multidimensional framework. This figure presents a non-exhaustive list of taxa as well as key references. Taxa in bold correspond to the images shown. Image credits (clockwise from top left): (threespine stickleback, Gasterosteus aculeatus) Travis Ingram; (thin-toed frog, Leptodactylus chaquensis) Raul Costa-Pereira; (wandering albatross, Diomedea exulans) Mark Jobling, licensed under public domain via Wikimedia Commons (https://commons.wikimedia.org/wiki/ File:070226_wandering_albatross_off_Kaikoura_3.jpg); (Eurasian perch, Perca fluviatilis) Richard Svanbäck; (sea otter, Enhydra lutris) "Mike" Michael L. Baird, licensed under CC BY 2.0 via Wikimedia Commons (https://commons.wikimedia.org/wiki/File: Sea-otter-morro-bay_13.jpg); (loggerhead sea turtle, Caretta caretta) U.S. National Oceanic and Atmospheric Administration (NOAA), licensed under public domain via Wikimedia Commons (https://commons.wikimedia.org/wiki/ File:Loggerhead_Sea_ turtle.jpg). 

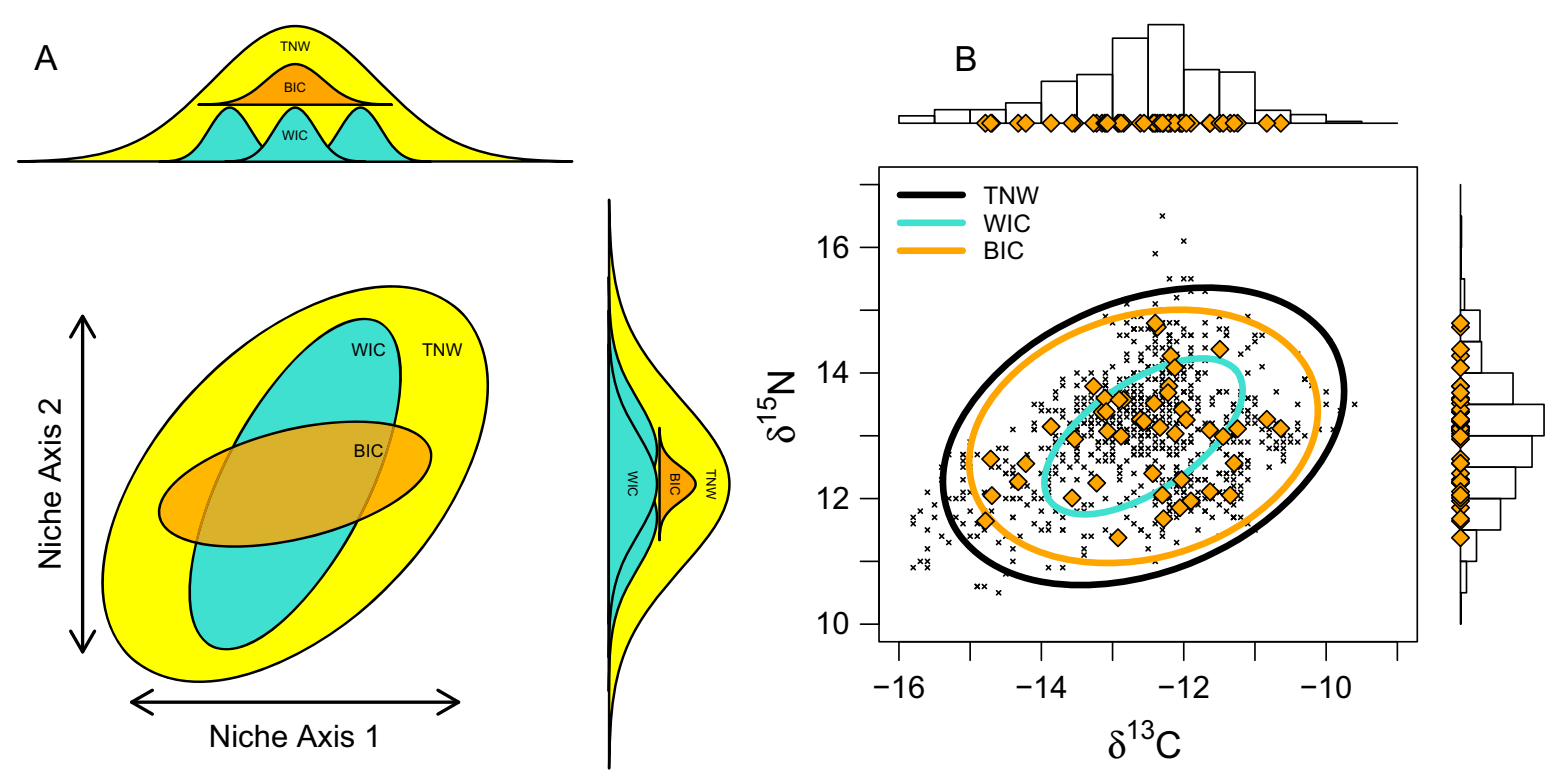

FIG. 2. (A) Translating the univariate representation of the BIC, WIC and TNW into multivariate ellipses. In this example, there is higher individual niche specialization (lower WIC/TNW) on niche axis 1 than niche axis 2, and consideration of both axes together reveals covariances in each component (with slightly different orientations). (B) Multivariate niche data for a sea otter (Enhydra lutris) population from Big Sur, California. 95\% confidence ellipses represent the niche components in two dimensions (stable isotopes $\delta^{13} \mathrm{C}$ and $\delta^{15} \mathrm{~N}$, serially sampled from vibrissae), while black symbols represent individual vibrissa segment isotope values and orange diamonds represent the individual means on the scatterplot and the histograms.

specialization in the population. These quantities are straightforward to define as variances in the case of a continuous niche axis such as prey size (Roughgarden 1972, 1974, Bolnick et al. 2002), or can be calculated using Shannon diversity indices as a proxy for variance when resource use is measured as frequencies of utilization of discrete food or habitat types (Roughgarden 1979, Bolnick et al. 2002). Alternative overlap-based measures of individual specialization are available for categorical data (Bolnick et al. 2002, Araújo et al. 2008), though these do not concurrently estimate population niche width. Measures of individual specialization based on resource categories typically do not consider quantitative differences between categories (e.g., prey size, nutritional value or trophic level), and can be influenced by the degree of taxonomic resolution (Newsome et al. 2015). To consider multiple, potentially covarying dimensions of resources use, we propose extending the analysis of the continuous niche components TNW, WIC and BIC into multiple ecological dimensions.

Much as the niche width of an individual or population can be measured as the variance of its resource use on a single continuous niche axis, so too can a multivariate niche width be defined based on variances and covariances of multiple niche axes. Given a suitable data set with repeated sampling of multiple niche dimensions for each individual (Box 1), we can extend the niche components TNW, WIC and BIC into matrix form. The $\mathrm{BIC}$ can be represented as an $m \times m$ matrix BIC in which the diagonal contains the variance among individual means for each of $m$ dimensions, while the off-diagonals represent covariances between individual means for pairs of niche dimensions. The BIC matrix can be interpreted as a phenotypic variance-covariance matrix $(\mathbf{P})$ in which the traits are niche axes, facilitating links to quantitative genetics (Box 2). A similar $m \times m$ matrix can be constructed for WIC, with the elements representing the average within-individual variances and covariances. The population niche width is then represented as the matrix $\mathbf{T N W}=\mathbf{W I C}+\mathbf{B I C}$. For example, given two niche axes $x$ and $y$ with multiple measurements $j$ for each individual $i$, the covariance matrix between all measurements in the population (TNW) can be written as the sum of the covariance matrix for the residuals of each observation against individual means (WIC) and the covariance matrix for individual means $\bar{x}$ and $\bar{y}$ (BIC):

$$
\operatorname{Cov}\left[x_{i, j}, y_{i, j}\right]=\operatorname{Cov}\left[x_{i, j}-\bar{x}_{i}, y_{i, j}-\bar{y}_{i}\right]+\operatorname{Cov}\left[\bar{x}_{i}, \bar{y}_{i}\right]
$$

The parameters of these matrices can be estimated using multiple response mixed models (Box 1). If data are approximately multivariate normal, each of these matrices can be visualized as a two-dimensional ellipse (Fig. 2) or a three-dimensional ellipsoid predicted to enclose a certain proportion of the multivariate distribution of resource use (e.g., $95 \%$, or approximately $40 \%$ for a standard ellipse; Jackson et al. 2011).

These covariance matrices can be used to ask questions about the size and what we refer to as the 'shape' of the niche components. The size of a niche component 


\section{Box 1: Data types and methodological considerations}

Analyses of individual specialization in multiple dimensions can incorporate a variety of data types, so long as they represent biologically meaningful niche dimensions informed by natural history knowledge of a particular study system. Perhaps most straightforward will be longitudinal data sets where each individual receives a relatively precise measure for each niche dimension (e.g., microhabitat or prey size) at each sampling period. Normality of data is not necessary for the calculation of covariance matrices, although for some applications either a (multivariate) normal distribution or some other distribution will need to be assumed. Depending on the scale of different measurements, standardization via z-scores or log transformation may make the niche component matrices more readily interpretable, though it is important to note that the choice of transformation will influence the shape of the inferred niche components. Partially discretized data may still provide information about continuous underlying niche axes; for example, dietary niche dimensions may be measured using mean sizes or trophic positions for multiple prey categories (taxon or functional groups), rather than measuring each item separately. The effectiveness of this approach will likely depend on the number of prey categories and their distribution in niche space, and simulations may be useful in understanding the effects of this aggregation. Consideration should also be given to the timescale over which niche data are collected: single cross-sectional samples (e.g., from stomach contents) are biased unless each sample captures a sufficient number of independent resource use decisions, while samples collected over very long timescales might misrepresent the degree of individual specialization occurring at any given time (Araújo et al. 2011, Novak and Tinker 2015).

A general method that can be used to analyze multivariate niche data is multiple response generalized linear mixed models (MGLMM). This flexible modeling approach can incorporate fixed and random effects, as well as different response variable distributions. Software capable of fitting these models includes the commercial software ASReml, which fits models with restricted maximum likelihood (Gilmour et al. 2009), and the MCMCglmm package in the R environment (Hadfield 2010, R Core Team 2014), which employs Bayesian Markov Chain Monte Carlo analysis. Individual identity can be modeled as a random effect to obtain either the restricted maximum likelihood or the posterior mean of the variances and covariances between individuals in each dimension (G-structure, BIC). The residual variances and covariances (R-structure) then quantify all variation left over after individual effects and any other terms are accounted for, and correspond to the average within-individual variation (WIC). The two matrices BIC and WIC can be added to estimate the total niche width (TNW). MGLMMs can incorporate other levels in hierarchical sampling designs - for example, if multiple diet items are measured for each measurement of habitat though to estimate the covariances in WIC it is important that at least some measurements of each niche axis are matched. A similar approach can be used to estimate and account for other forms of intraspecific niche variation (e.g., due to age class, sex, or discrete morph) by including them as additional fixed effects in the model.

Visualization and interpretation of multivariate model fits will be simplest if the data are approximately multivariate normally distributed, and niche components can be displayed as ellipses or ellipsoids (Jackson et al. 2011, Swanson et al. 2015). However, MGLMMs can incorporate other data distributions, such as binomial or threshold models for binary data, and individual traits may be inferred on a latent scale. The development of methods for measuring standard statistical properties such as repeatability (Nakagawa and Schielzeth 2010) and power (Johnson et al. 2015) are ongoing for GLMs, so some validation is likely to be needed for analyses of individual specialization combining data distributions into multivariate measures of niche components.

A variety of hypotheses related to multivariate niche variation can be tested using MGLMMs. The fit of competing models can be compared using likelihood ratios or information criteria to assess support for a niche component- for example, to test for non-zero individual variance or covariance terms. A similar approach can test whether groups including age classes, sexes, or different species have the same or different WICs or BICs. Related hypotheses about the proportionality of matrices (e.g., is the BIC of a population following competitive release a scaled-up version of initial BIC) do not appear to be implemented in currently available MGLMM software, but should be straightforward extensions. As many of these methods are still in development, we recommend analyzing simulated or resampled data where possible to confirm the expected properties of the niche components in the absence of some process of interest.

can be measured as the sum of the eigenvalues of the corresponding matrix; this may be more interpretable if variables measured on different scales are first scaled to produce z-scores. Analogous to the univariate metric WIC/TNW, the size of the WIC divided by the size of the TNW provides a multivariate measure where lower 


\section{Box 2: Multivariate niche variation and quantitative genetics}

Studies of multidimensional individual specialization can draw from the sizeable literature dealing with quantitative genetic variation and evolution of traits controlled by the combined action of many genes of small effect. Generalization of the breeder's equation permitted the use of matrix algebra to study genetic variation and response to selection in multiple correlated traits (Lande and Arnold 1983), and quantitative genetics has subsequently developed a rich set of methods for considering both genetic and environmental factors that influence evolutionary outcomes (Schluter 1996, Phillips and Arnold 1999, Steppan et al. 2002, Arnold et al. 2008). Several aspects of this work, particularly the study of G-matrices of additive genetic variation and covariation underlying traits, are applicable to our framework for studying multivariate niche variation.

The most straightforward such link is the fact that the BIC is in fact a specific type of phenotypic variancecovariance matrix (P-matrix) in which the traits are ecological dimensions such as diet or habitat use. This implies that $\mathbf{B I C}=\mathbf{G}+\mathbf{E}$ : between-individual variation is determined by some combination of additive genetic (co)variation and environmental (co)variation. Therefore, the population niche $\mathbf{T N W}=$ $\mathbf{G}+\mathbf{E}+\mathbf{W I C}$, adding within-individual variation to the conventional partitioning of phenotypic variation. We might anticipate that the genetic component will be low for traits often defined by flexible behavior and ecological interactions, but this is a hypothesis that should be tested when possible, as traits related to behavior (Box 3), environmental tolerance and resource use can have a substantial genetic basis (Mousseau and Roff 1987, Robinson and Page 1989).

It is therefore possible in principle to estimate quantitative genetic parameters for BIC, so long as a suitable combination of genetic and ecological data can be obtained. Either wild populations with pedigrees, or laboratory crosses released into a natural or semi-natural environment, could be used to infer the size and shape of the G-matrix relative to BIC and TNW. Quantitative trait locus mapping or genome-wide associations studies could be used to identify regions of the genome associated with different niche dimensions, and knowledge of the genetic architecture underlying niche variation may be valuable in predicting population responses to selection imposed by ecological change.

values represent stronger overall individual specialization. The relative size of each component can be visualized by comparing the ellipses (or ellipsoids) described above, though unlike the matrices themselves, the areas (or volumes) are not precisely additive. The shape of a niche component encompasses the variances of each niche axis along the diagonal of the matrix, as well as the covariances in the off-diagonal. Ecologically interesting properties of the shape of these matrices include their eccentricity (relative dominance of the first eigenvalue) and orientation (rotation of the matrix as defined by its eigenvectors). Measurement of the size and shape of the covariance matrices representing niche components can be used to test hypotheses about the causes and consequences of multidimensional individual specialization.

\section{IMPLICATIONS OF MULTIDIMENSIONAL INDIVIDUAL SPECIALIZATION}

In the following section we outline how individual specialization in multiple ecological dimensions has the potential to alter our understanding of a range of ecological and evolutionary processes. We build on a recent review of the consequences of intraspecific variation (Bolnick et al. 2011), and focus on the specific impacts that individual specialization in a multidimensional ecological niche space can have on processes in ecology and evolutionary biology.

\section{Coexistence and community structure}

Community ecology has been transformed by a modern understanding of the conditions for species coexistence over the past two decades (Chesson 2000), and recent studies have begun to consider the impact of intraspecific variation on coexistence and community assembly (Clark 2010, Violle et al. 2012, Barabás and D'Andrea 2016, Hart et al. 2016). However, these approaches have so far either considered only one niche axis at a time, or have considered multiple niche axes but not partitioned variation into between- and within-individual components. In this section we discuss how considering the dimensionality of between-individual and within-individual niche variation can yield new insights into the mechanisms responsible for coexistence and community assembly.

Recent theory states that the possibility for stable coexistence between species depends on the magnitude of both niche differences and fitness inequalities between them (Chesson 2000, Adler et al. 2013). Niche differentiation between species can be a stabilizing process by causing the average magnitude of intraspecific competition to exceed that of interspecific competition. The extent of niche overlap should thus be correlated with the strength of stabilizing processes favoring coexistence. As species compete simultaneously and interactively for different types of resources (e.g., microhabitats and prey 
types), the true multivariate niche overlap can depend on both the dimensionality of the niche and the shape of niche components. In this context, the possibility of quantifying variation both between and within individuals of competing species in multiple dimensions (Fig. 3) is a powerful advantage of our framework, allowing more meaningful measurement of niche overlap.

Both the between- and within-individual components of the population's niche will determine the true niche overlap between species, but in practice niche overlap is often calculated without considering within-individual variation. For example, a popular use of stable isotope data (e.g., $\delta^{15} \mathrm{~N}$ and $\delta^{13} \mathrm{C}$ ) is to infer niche overlap of multiple species in a community. However, most isotope studies rely on a single sample per individual, and if the tissue analyzed has a relatively slow turnover rate (such as muscle), it will largely indicate only the BIC of the population's total niche width (as well as various sources of error) and neglect the WIC. It may be possible to estimate the WIC if multiple isotope samples can be obtained per individual, either by serially sampling inert tissues such as vibrissae or by sampling multiple tissues that vary in turnover time and integrate diet over different time scales (Fig. 2B; Newsome et al. 2009, Matich et al. 2011). However, even in these cases we note that a substantial part of the within-individual variation is likely to be missed, as each tissue sample will still represent an average over multiple diet items consumed over the relevant time scale. In the more common scenario of a single sample per individual, niche estimates incorporating primarily the BIC can be expected to underestimate niche overlap, especially in species in which WIC is large relative to TNW, or WIC and BIC have very different shapes. As an example, we show how adding repeated measures of multiple niche dimensions for each individual (i.e.,, incorporating WIC) can increase the perceived niche overlap between congeneric thin-toed frog species (Leptodactylus spp.) in the Pantanal wetlands of Brazil (details in Appendix S1). In this system, we can obtain a more meaningful measure of niche overlap when multiple niche dimensions (prey $\delta^{13} \mathrm{C}$ and prey size) are measured together, and when the WIC (based on multiple samples in each frog's gut contents) is considered along with the BIC (Fig. 3).

While improved measurement of niche overlap is important, the degree and shape of individual niche variation also have the potential to impact species coexistence. Recent theory predicts that individual variation in a single dimension should impede coexistence by causing individuals to compete more strongly with ecologically similar heterospecifics than with dissimilar conspecifics (Barabás and D’Andrea 2016, Hart et al. 2016). Other theoretical work emphasizes the role of individual differences in responses to environmental variation (Clark et al. 2007, 2010, 2011), or species differences in the shape of nonlinear functional responses (Armstrong and McGehee 1980). In general, we can predict that species coexistence is more likely if species differ not only in niche position but also in the shape of niche variation, meaning they will respond differently to multiple environmental variables. Species' niche components can be compared in our framework: multiple response GLMMs can model shared or different niche components for each
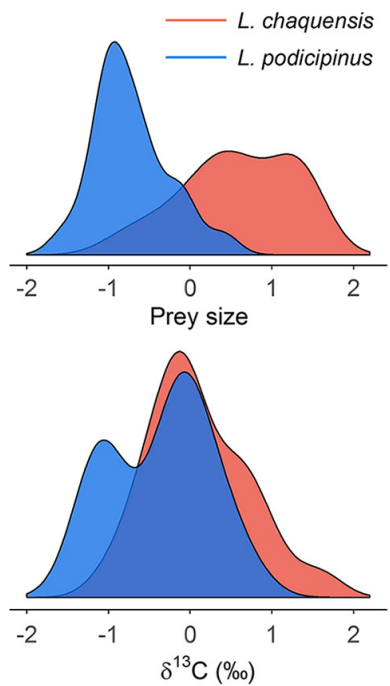

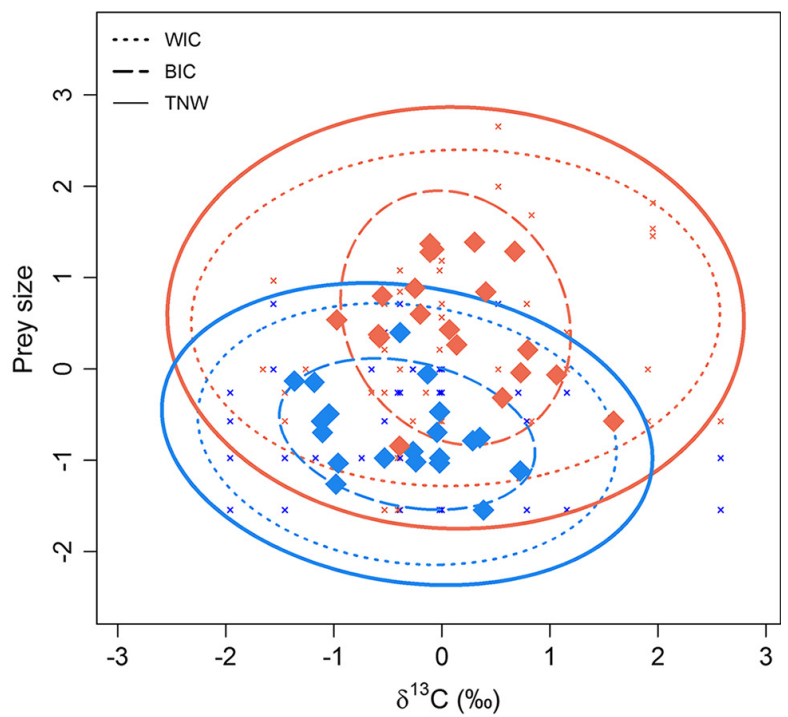

FIG. 3. Illustration of how the dimensionality of niche variation may relate to perceived measures of niche overlap between two congeneric frog species (Leptodactylus spp.) from the Pantanal wetlands, Brazil. When examined one niche axis at a time, the two species appear to have relatively high overlap in relation to $\delta^{13} \mathrm{C}$, but not to prey size (left panels). Considering both niche axes simultaneously, niches can be represented as $95 \%$ confidence standard ellipses for the BIC, WIC and TNW. Species show low overlap when only the BIC is examined (dotted lines, as is usually the case for stable isotope niche data), but in fact the overlap is considerably high when WIC is considered (solid lines). Crosses represent the position of each prey found in gut contents and diamonds represent the individual frogs' means of prey size and $\delta^{13} \mathrm{C}$. 
species, and methods for matrix comparison (Phillips and Arnold 1999, Steppan et al. 2002) could be adapted to test for similarity in the shape of the WIC or BIC. We could therefore extend hierarchical approaches to traitbased ecology (Violle et al. 2012) by partitioning the community niche structure into variation between species, between individuals and within individuals.

\section{Food web and ecosystem processes}

Intraspecific variation in one or more niche dimensions has the potential to modify a species' impact on other trophic levels, and on broader food web and ecosystem functions. The diversity of discrete genotypes in clonal species can have ecosystem-scale consequences analogous to the effects of species diversity on ecosystem function (Whitham et al. 2006, Hughes et al. 2008), and continuous variation among individuals can be expected to have many of the same effects (Bolnick et al. 2011, Moran et al. 2016). One significant mechanism by which intraspecific variation can alter a species' contribution to ecological processes can be explained by a mathematical expression known as Jensen's inequality (Jensen 1906, Ruel and Ayres 1999, Moran et al. 2016). For a process that is a concave function of a trait or niche measure that varies among individuals, the mean function value of all individuals will be lower than the function expressed at the population mean trait value (while the reverse is true for convex functions). Thus, individual niche variation should decrease the population-level outcome for concave functions such as saturating functional responses, thermal performance curves or unimodal relationships between a resource axis and resource productivity (Martin and Huey 2008, Denno and Lewis 2009, Bolnick et al. 2011). These effects should translate into multiple dimensions when the process of interest varies as a concave (or convex) function of multiple trait or niche dimensions (Madansky 1959), but detailed theoretical explorations of the consequences of Jensen's inequality in multiple dimensions are rare (see, however, Evans et al. 2013). To illustrate the potential effects, we used a set of simulations where performance is a strictly concave function of multiple ecological traits. We show that the magnitude by which intraspecific variation reduces population mean performance can increase with niche dimensionality, total niche width, and the extent of individual specialization (Fig. 4, details in Appendix S1). A full theoretical treatment will be needed to clarify these predictions and to evaluate how the effect is influenced by the covariance between niche axes or by
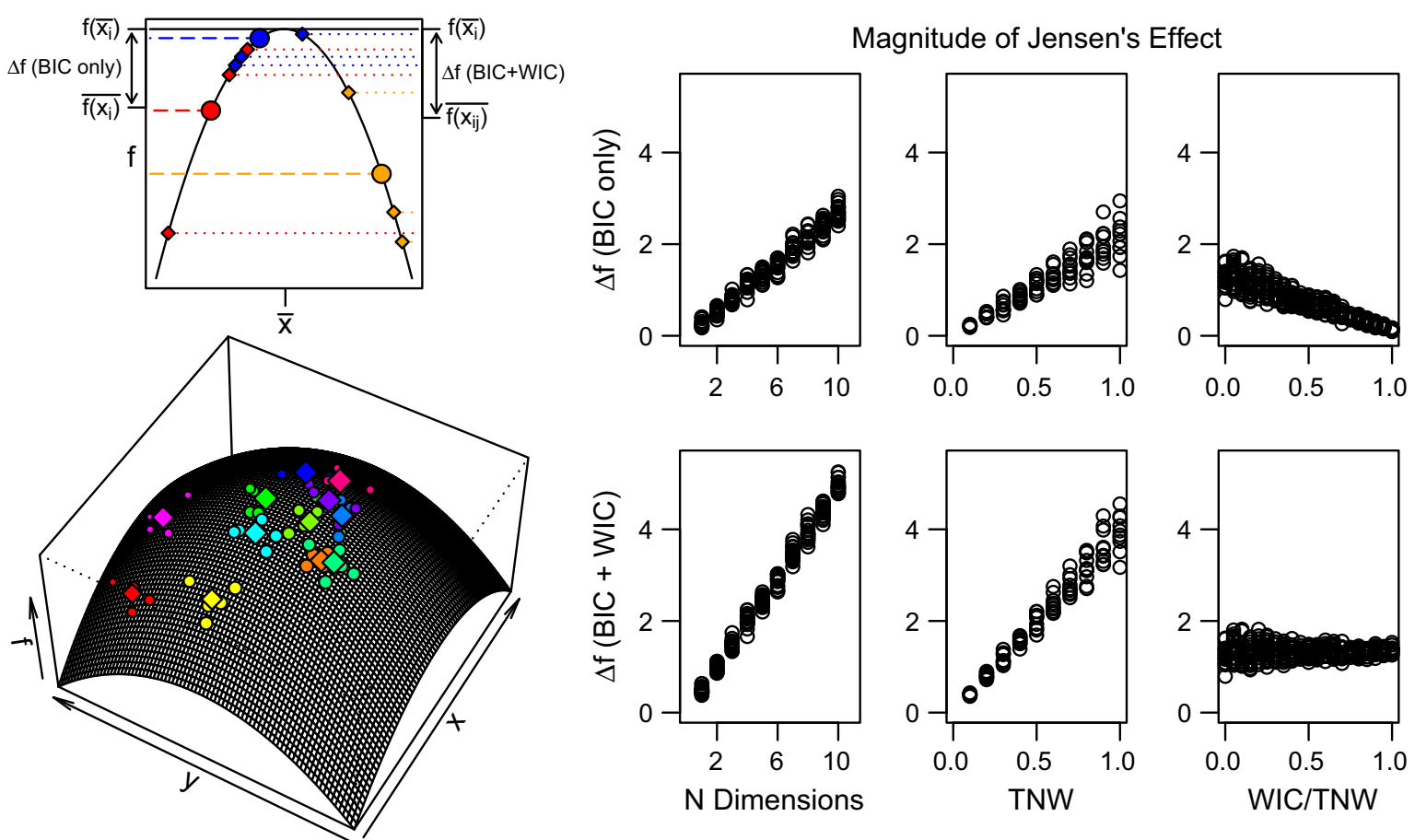

FIG. 4. Jensen's inequality in multiple dimensions. Top left: for a concave function $f$ of a trait $x$, the mean individual function will be less than the function evaluated at the mean trait value if individuals vary in $x$ (left axis), and the mean function will be further depressed if within-individual variation in $x$ is accounted for (right axis). Individual means are represented by colored circles and within-individual variation is represented by diamonds of the same color. Bottom left: illustration of a function (quadratic) that is concave in two dimensions, with symbols as in the one-dimensional panel. Panels on right: the magnitude of the decrease in the value of a strictly concave function due to Jensen's inequality depends on the number of dimensions, the total niche width (TNW, given two dimensions), and the degree of individual specialization (given two dimensions and constant TNW). 
mixtures of convex and concave functions. Experimental manipulations of the degree of individual variation in multiple traits may then reveal whether the expected effects of multidimensional niche variation are evident in empirical systems.

Another scenario in which the dimensionality of niche variation may have particular importance is when diet diversity encompasses both "horizontal" and "vertical" dimensions of food webs. The horizontal diversity refers to the variety of species within a trophic level, potentially encompassing energy pathways supported by multiple primary producers, while vertical diversity refers to variation across trophic levels or in trophic position (Matthews et al. 2010). Empirical evidence indicates that intraspecific competition in a consumer can drive population niche expansion via increased between-individual variation (Svanbäck and Bolnick 2007, Araújo et al. 2011, Tinker et al. 2012, Jones and Post 2016), but the degree to which this process impacts lower trophic levels could depend on the dimension in which niche expansion is most pronounced. One possibility is that niche expansion will occur via increased horizontal diet variation such that all individuals specialize on prey at the same trophic level. In this case, the total strength of topdown control may be enhanced due to the increased consumer population size, even if the per-capita impact is lower because the impact is spread across more trophic links (Bolnick et al. 2011). In contrast, if niche expansion involves increased vertical diet variation (omnivory) and individuals specialize on prey at different trophic levels, top-down control may be dampened (Bruno and O'Connor 2005) despite the increase in consumer density. A related phenomenon is the coupling of distinct food webs via horizontal diet diversity of top predators, which under some conditions is predicted to stabilize the larger system (McCann et al. 2005, Rooney et al. 2006). Horizontal niche variation will alter the degree of coupling if individuals specialize on distinct energy sources (e.g., pelagic vs. littoral; Quevedo et al. 2009), whereas vertical niche variation may not affect the degree of coupling. Knowledge of the extent and shape of individual specialization in multiple dimensions may therefore allow us to predict qualitatively different trophic dynamics and ecosystem processes.

\section{Niche shifts and ecological speciation}

Thus far we have focused on potential effects of multidimensional individual niche variation that do not necessarily involve a change in the niche position of the focal species. Of course, many important questions in ecology and evolution concern shifts in the population mean niche position: single populations may undergo niche shifts in response to biotic or abiotic environmental changes, while speciation often involves the evolution of distinct niches in newly formed species. We argue that given the natural links between quantitative genetic theory and multidimensional individual specialization
(Box 2), consideration of the dimensionality of niche variation will enrich our understanding of the ecological and evolutionary dynamics of niches.

A shift in the average niche position of a population will involve some combination of evolutionary change and phenotypic plasticity. When niche variation among individuals is partially heritable, selective pressures such as biotic interactions may drive an evolutionary shift in the niche position. For example, competition and predation by an intraguild predator, prickly sculpin (Cottus asper), have led threespine stickleback (Gasterosteus aculeatus) to undergo an evolutionary shift toward increased use of zooplankton and open water habitats (Ingram et al. 2012, Miller et al. 2015). A byproduct of predator-induced life history evolution in Trinidadian guppies (Poecilia reticulata) is a shift by guppies in highpredation environments to feed at a higher trophic level (greater reliance on invertebrates than algae) than populations in low-predation environments (Zandona et al. 2011). Finally, the native lizard Anolis carolinensis has undergone an evolutionary shift to use more arboreal microhabitats following the invasion of Florida by its congener A. sagrei (Stuart et al. 2014). Other niche shifts can occur without evolutionary change, where species with flexible foraging and habitat selection behaviors alter their resource use within a generation. Short term (e.g., within-generation) responses to the presence of predators or competitors have been shown for both the niche position of a focal species (Vander Zanden et al. 1999, Eklöv and Svanbäck 2006) and for the degree of niche variation among individuals (Bolnick et al. 2010).

We propose that the dimensionality of individual variation may bias the direction of niche shifts in response to biotic or abiotic changes. When a species undergoes an evolutionary response to selection on a heritable trait, a well-established body of theory and empirical study shows that the dimensionality and orientation of the G-matrix can bias the direction of phenotypic evolution along 'genetic lines of least resistance' (Schluter 1996, McGuigan et al. 2005, Hansen and Houle 2008, Dochtermann and Dingemanse 2013). If genetic variation underlies between-individual niche variation (Box 2), we can therefore predict that ecological niche shifts will be biased in the direction of greatest genetic variation. It is also conceivable that the shape of niche variation can bias the direction of niche shifts that occur via behavioral flexibility or other forms of phenotypic plasticity, even in the absence of evolutionary change (Box 3). If individuals exhibit flexibility that allows shifts toward novel or underutilized resources, this flexibility may manifest as the WIC within a population prior to the niche shift. The shape of the WIC might influence the direction of the niche shift if it reflects constraints on the extent of plasticity, where only particular regions of niche space can be readily explored by an individual. In contrast, if niche shifts occur as individuals learn from the resource acquisition behavior of more successful individuals, the type of individuals available to imitate should be determined by 


\section{Box 3: Multivariate niche variation and behavior}

Appreciation for the importance of between-individual variation has also increased in behavioral ecology. Our framework shares similarities with methods used in the study of repeatable individual differences in behavior (Dall et al. 2012). These differences, sometimes called animal personalities, manifest as behavioral syndromes when individuals vary in multiple correlated behaviors. For example, a proactive behavioral syndrome is associated with positive covariance between boldness, activity, and aggression. Behavioral ecologists have applied statistical methods including (generalized) linear mixed models to partition behavioral variation within vs. among individuals (Dingemanse and Dochtermann 2013).

In addition to methodological similarities, multidimensional behavior and multidimensional niche variation may be linked directly when behavioral variation underlies resource selection. For example, bolder individuals are predicted to use habitats farther from shelter and to forage on prey that expose the individual to greater predation risk. Early work on behavioral variation in fishes examined the use of sheltered nearshore habitats vs. exposed open water habitats, aligning the position of an individual on the bold-shy personality axis with its use of specific habitats and prey types (Wilson et al. 1993). In fact, variables such as time spent in exposed vs. sheltered habitats are measures of resource (microhabitat) use that also serve as proxies for personality traits. Despite these links, the same individuals are rarely measured for both behavioral and niche data, particularly in field conditions. Paired measures of behavior and resource use for the same individuals could be incorporated into a larger multivariate analysis to examine linkages between personality and individual specialization.

Examination of behavioral variation may also help us to interpret the within-individual component of niche variation. As outlined in our discussion of niche shifts, the orientation of the WIC might bias the direction of a niche shift if this variation reflects the extent of behavioral flexibility of each individual. In the framework we have presented, the WIC is treated as a population-level covariance matrix estimated using the average of each individual's variation and covariation in resource use. However, individuals with greater exploratory behavior or activity levels may be more likely to sample novel prey items, potentially leading to between-individual variation in the degree of within-individual variation (Cleasby et al. 2015). While this form of heterogeneity among individuals is missed when WIC is calculated as an average, it can be incorporated into hierarchical models if variation in individual niche width is of interest.

the size and shape of the BIC. These scenarios should lead to rather different predictions if the WIC and BIC have very different orientations. We therefore propose the novel hypothesis that the trajectory of a niche shift may be influenced by the shape of multidimensional niche variation, and be biased toward the 'ecological lines of least resistance' represented in the multivariate niche components WIC and/or BIC (Fig. 5).
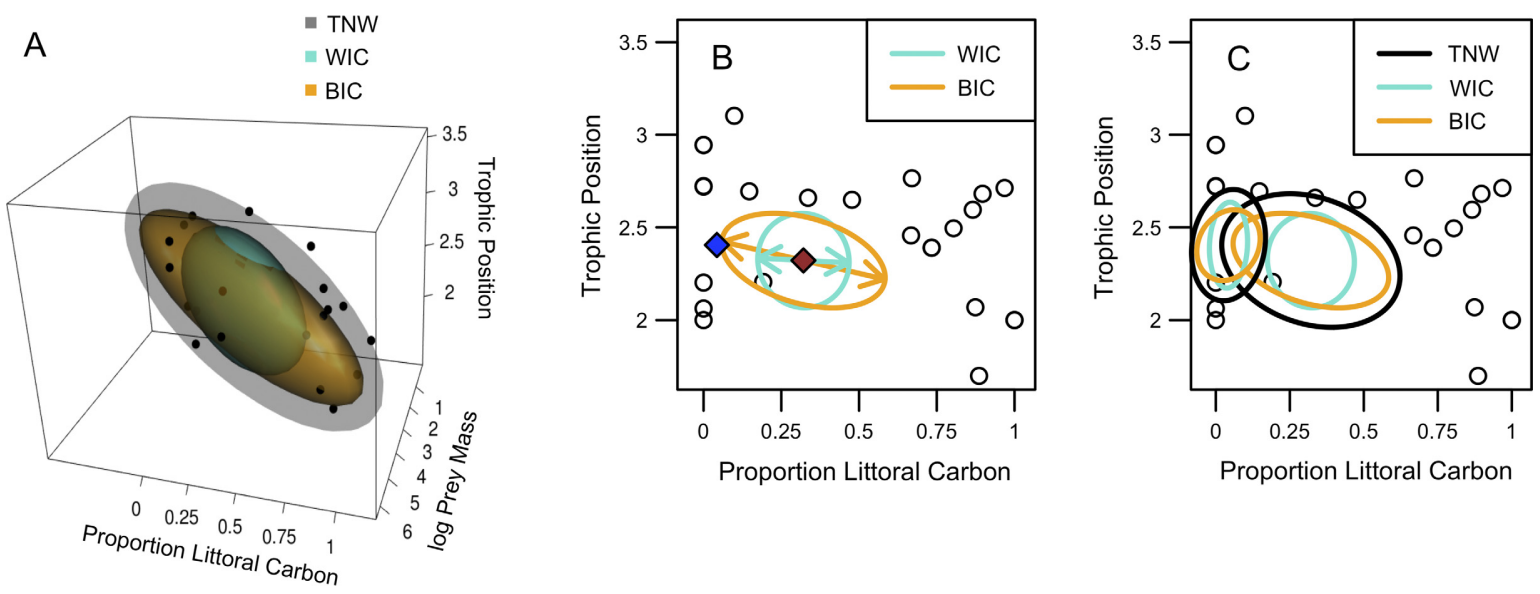

FIG. 5. Multidimensional diet variation in threespine stickleback. (A) three-dimensional niche variation in the proportion of littoral carbon, trophic position, and prey size, represented by standard ellipsoids for a population with both littoral and pelagic prey bases. (B) Niche components BIC and WIC represented as standard ellipses for the first two dimensions for the same population, with the direction of greatest variation indicated by arrows and the direction of the niche shift (to the "pelagic" enclosed population type) indicated by the blue symbol. (C) Niche components TNW, BIC and WIC for both population types. 
To illustrate how niche shifts may align with the shape of niche variation, we analyze data from a field experiment using enclosed populations of threespine stickleback (details in Appendix S1). Stickleback individuals vary in at least two dietary niche dimensions, trophic position and use of pelagic vs. littoral-derived carbon (Matthews et al. 2010, Arnegard et al. 2014). We manipulated habitat availability by building enclosures in habitats with access to littoral and pelagic prey typical of small lakes, as well as enclosures with restricted access to the benthic substrate. This manipulation effectively imposed a niche shift, and the direction of this shift aligned with the direction of greatest between-individual variation in the initial population. The niche shift also resulted in differences in the size and shape of the niche components between populations (Fig. 5, Appendix S1).

These predictions are also relevant for the study of ecological speciation, which occurs when reproductive isolation results in some way from divergent natural selection acting on the ecological niche (Schluter 2009, Nosil 2012). Ecological speciation involves a niche shift by one or both incipient species, and when the niche shift is multidimensional our hypotheses about the influence of the shape of niche components come into play. For example, differences between sympatric stickleback species also tend to align with the direction of greatest between-individual variation in single-species lakes (Matthews et al. 2010). There is theoretical and empirical support for the idea that when multifarious divergent selection acts simultaneously on multiple traits or niche dimensions, speciation is more likely to proceed to completion than when only a single trait is under selection (Rice and Hostert 1993, Nosil et al. 2009, Nosil 2012). This effect can result from increased total strength of divergent selection, or by dimensionality per se if selection affects a greater proportion of the genome (Rice and Hostert 1993, Nosil and Sandoval 2008). The number of dimensions in which individuals vary is also predicted to increase the likelihood that some aspect of this variation will be under disruptive selection (Doebeli and Ispolatov 2010), so the shape and size of the multivariate niche components might influence the probability of speciation. Given the central role of individual variation in models of ecological speciation and the likely multifarious selection imposed by divergence on environmental gradients such as water depth or elevation (Doebeli and Dieckmann 2003), understanding the dimensionality of individual specialization should improve our ability to predict when speciation will or will not occur.

\section{Conclusions and Future Directions}

The concept of the niche is central to many ideas in ecology and evolutionary biology, and it is now apparent that measurement of niches at the population level often neglects important resource use variation at the individual level. While many niche concepts incorporate multiple ecological dimensions, little consideration has been given to the dimensionality of individual ecological variation. We have shown how a framework for thinking about individual niches in multiple dimensions can enhance our understanding of a range of ecological and evolutionary concepts, and presented testable hypotheses based on this framework. We conclude by outlining some areas of further research that will help to place the idea of multidimensional individual specialization within a broader understanding of ecology and evolution.

As the niche components TNW, BIC and WIC have not been measured for most species, more data must be collected before we can identify generalities in the structure of multidimensional niche variation. Given more such measures, we can test whether the size and shape of niche components vary among taxa or ecosystems. Methods for comparing sets of variance-covariance matrices, such as eigentensor analysis (Aguirre et al. 2014) could be used to identify regularities in the structure of niche components. We could also analyze niche variation in an explicitly phylogenetic context (Maldonado et al. 2017), extending our hypotheses about niche shifts to multiple related species. If evolutionary divergence between species is constrained or guided by the available intraspecific variation, the matrix of divergence between species is predicted to align with intraspecific phenotypic (P or BIC; see Box 2) or genetic (G) variance-covariance matrices (Felsenstein 1988). We could use a similar approach to test whether amongspecies divergence can be predicted by the shape of either between- or within-individual ecological variation. In systems with substantial ecological variation among related species, it should be possible to assess the evolutionary history and the current diversity of multivariate niche components, which may then have implications for ecosystem function and coexistence.

For individual specialization to have a significant influence on either evolutionary or population dynamics, it should bear a relationship with some component of organismal fitness. Directional, stabilizing and disruptive natural selection has been measured on a wide range of morphological traits (Kingsolver et al. 2001) and numerous studies have evaluated the fitness consequences of behavioral variation among individuals (Dingemanse and Réale 2005, Smith and Blumstein 2008, Ballew et al. 2017), but attempts to link fitness to more direct measures of the ecological niche are much rarer (Bolnick and Araújo 2011). While the often messy nature of ecological data presents challenges in precisely measuring multivariate selection surfaces, the direct link between resource use and many ecosystem processes makes this a worthwhile goal. Similarly, understanding how multidimensional niche variation relates to the absolute fitness of a species (its population growth) and its relative competitive ability should enhance our understanding of population and community dynamics.

A key issue going forward will be integrating this perspective on multidimensional individual specialization with modern coexistence theory, perhaps by linking 
measures of multivariate niche overlap to the strength of stabilizing mechanisms (Letten et al. 2016). Further, a reconciliation of the classical niche theory extended in our multidimensional framework with the treatment of 'niche factors' in many modern works (Chase and Leibold 2003) will need to be attempted. Individual variation in the use of different food sources and habitats is unlikely to be independent of measures such as the $\mathrm{R} *$ that predict population-level resource consumption, though recent theory suggests that individual variation in either univariate resource use or demographic parameters should make coexistence of competitors less likely (Barabás and D’Andrea 2016, Hart et al. 2016). We acknowledge that our framework does not incorporate all current conceptions of the ecological niche, and stress the importance of a broader consideration of the dimensionality of individual niche variation.

Finally, in addition to cataloguing the incidence of individual specialization that encompasses multiple ecological dimensions, both the causes and consequences of multidimensional niche variation will need to be explored in more detail. Experimental studies of the potential drivers of shifts in the extent or shape of niche variation are likely to prove fruitful in identifying the causal factors and assessing how rapidly niche components change. It is not yet certain to what extent aspects of the shape of niche variation such as orientation or eccentricity may impact ecological or evolutionary processes, relative to the niche position or the overall degree of niche variation. Theoretical work in this direction, coupled with direct manipulation of the dimensionality of variation among individuals in suitable systems, should help us to understand the importance of the dimensionality of individual niche variation.

\section{ACKNOWLEDGMENTS}

We thank D. Bolnick, J. Jandt, M. Schofield, and members of Travis Ingram's research group for comments on this manuscript and discussion of the ideas within it. This work was funded by the Royal Society of New Zealand Marsden Fund (UoO1409), and RC-P was supported by grants \#2014/ 20924-5 and \#2016/09038-9, São Paulo Research Foundation (FAPESP).

TI proposed the manuscript topic; TI and RCP carried out the analyses for the case studies; TI and MSA wrote the first draft of the manuscript collaboratively, and TI, RCP and MSA contributed to revisions.

\section{Literature Cited}

Ackerly, D. D., and W. K. Cornwell. 2007. A trait-based approach to community assembly: partitioning of species trait values into within- and among-community components. Ecology Letters 10:135-145.

Adler, P. B., A. Fajardo, A. R. Kleinhesselink, and N. J. B. Kraft. 2013. Trait-based tests of coexistence mechanisms. Ecology Letters 16:1294-1306.

Aguirre, J. D., E. Hine, K. McGuigan, and M. W. Blows. 2014. Comparing G: multivariate analysis of genetic variation in multiple populations. Heredity 112:21-29.
Araújo, M. S., P. R. Guimarães, R. Svanbäck, A. Pinheiro, S. F. dos Reis, and D. I. Bolnick. 2008. Network analysis reveals contrasting effects of intraspecific competition on individual vs. population diets. Ecology 89:1981-1993.

Araújo, M. S., D. I. Bolnick, and C. A. Layman. 2011. The ecological causes of individual specialisation. Ecology Letters 14:948-958.

Armstrong, R. A., and R. McGehee. 1980. Competitive exclusion. American Naturalist 115:151-170.

Arnegard, M. E., et al. 2014. Genetics of ecological divergence during speciation. Nature 511:307-311.

Arnold, S. J., R. Bürger, P. A. Hohenlohe, B. C. Ajie, and A. G. Jones. 2008. Understanding the evolution and stability of the G-Matrix. Evolution 62:2451-2461.

Ballew, N. G., G. G. Mittelbach, and K. T. Scribner. 2017. Fitness consequences of boldness in juvenile and adult largemouth bass. American Naturalist 189:396-406.

Barabás, G., and R. D'Andrea. 2016. The effect of intraspecific variation and heritability on community pattern and robustness. Ecology Letters 19:977-986.

Baylis, A. M. M., R. A. Orben, J. P. Y. Arnould, K. Peters, T. Knox, D. P. Costa, and I. J. Staniland. 2015. Diving deeper into individual foraging specializations of a large marine predator, the southern sea lion. Oecologia 179:1053-1065.

Bolnick, D. I., and M. S. Araújo. 2011. Partitioning the relative fitness effects of diet and trophic morphology in the threespine stickleback. Evolutionary Ecology Research 13: 439-459.

Bolnick, D., L. Yang, J. A. Fordyce, and J. Davis. 2002. Measuring individual-level resource specialization. Ecology 83:2936-2941.

Bolnick, D. I., R. Svanbäck, J. A. Fordyce, L. H. Yang, J. M. Davis, C. D. Hulsey, and M. L. Forister. 2003. The ecology of individuals: incidence and implications of individual specialization. American Naturalist 161:1-28.

Bolnick, D. I., T. Ingram, W. E. Stutz, L. K. Snowberg, O. L. Lau, and J. S. Paull. 2010. Ecological release from interspecific competition leads to decoupled changes in population and individual niche width. Proceedings of the Royal Society B: Biological Sciences 277:1789-1797.

Bolnick, D. I., P. Amarasekare, M. S. Araújo, R. Bürger, J. M. Levine, M. Novak, V. H. W. Rudolf, S. J. Schreiber, M. C. Urban, and D. A. Vasseur. 2011. Why intraspecific trait variation matters in community ecology. Trends in Ecology \& Evolution 26:183-192.

Brodersen, J., H. J. Malmquist, F. Landkildehus, T. L. Lauridsen, S. L. Amsinck, R. Bjerring, M. Søndergaard, L. S. Johansson, K. S. Christoffersen, and E. Jeppesen. 2012. Short-and long term niche segregation and individual specialization of brown trout (Salmo trutta) in species poor Faroese lakes. Environmental Biology of Fishes 93:305-318.

Bruno, J. F., and M. I. O'Connor. 2005. Cascading effects of predator diversity and omnivory in a marine food web. Ecology Letters 8:1048-1056.

Casper, R. M., M. D. Sumner, M. A. Hindell, N. J. Gales, I. J. Staniland, and S. D. Goldsworthy. 2010. The influence of diet on foraging habitat models: a case study using nursing Antarctic fur seals. Ecography 33:748-759.

Ceia, F. R., R. A. Phillips, J. A. Ramos, Y. Cherel, R. P. Vieira, P. Richard, and J. C. Xavier. 2012. Short-and long-term consistency in the foraging niche of wandering albatrosses. Marine Biology 159:1581.

Chase, J. M., and M. A. Leibold. 2003. Ecological niches: linking classical and contemporary approaches. University of Chicago Press, Chicago, Illinois, USA.

Chesson, P. 2000. Mechanisms of maintenance of species diversity. Annual Review of Ecology, Evolution, and Systematics 31:343-366. 
Chilvers, B. L. 2008. Foraging site fidelity of lactating New Zealand sea lions. Journal of Zoology 276:28-36.

Chilvers, B. L., and I. S. Wilkinson. 2009. Diverse foraging strategies in lactating New Zealand sea lions. Marine Ecology Progress Series 378:299-308.

Clark, J. S. 2010. Individuals and the variation needed for high species diversity in forest trees. Science 327:1129-1132.

Clark, J. S., M. Dietze, S. Chakraborty, P. K. Agarwal, I. Ibanez, S. LaDeau, and M. Wolosin. 2007. Resolving the biodiversity paradox. Ecology Letters 10:647-659.

Clark, J. S., et al. 2010. High-dimensional coexistence based on individual variation: a synthesis of evidence. Ecological Monographs 80:569-608.

Clark, J. S., D. M. Bell, M. H. Hersh, M. C. Kwit, E. Moran, C. Salk, A. Stine, D. Valle, and K. Zhu. 2011. Individual-scale variation, species-scale differences: inference needed to understand diversity. Ecology Letters 14:1273-1287.

Cleasby, I. R., S. Nakagawa, and H. Schielzeth. 2015. Quantifying the predictability of behaviour: statistical approaches for the study of between-individual variation in the within-individual variance. Methods in Ecology and Evolution 6:27-37.

Cucherousset, J., A. Acou, S. Blanchet, J. R. Britton, W. R. C. Beaumont, and R. E. Gozlan. 2011. Fitness consequences of individual specialisation in resource use and trophic morphology in European eels. Oecologia 167:75-84.

Dall, S. R. X., A. M. Bell, D. I. Bolnick, and F. L. W. Ratnieks. 2012. An evolutionary ecology of individual differences. Ecology Letters 15:1189-1198.

Denno, R. F., and D. Lewis. 2009. Predator-prey interactions. Pages 202-212 in S. A. Levin, editor. The Princeton guide to ecology. Princeton University Press, Princeton, New Jersey, USA.

Dieckmann, U., and M. Doebeli. 1999. On the origin of species by sympatric speciation. Nature 400:354-357.

Dingemanse, N. J., and N. A. Dochtermann. 2013. Quantifying individual variation in behaviour: mixed-effect modelling approaches. Journal of Animal Ecology 82:39-54.

Dingemanse, N. J., and D. Réale. 2005. Natural selection and animal personality. Behaviour 142:1159-1184.

Dochtermann, N. A., and N. J. Dingemanse. 2013. Behavioral syndromes as evolutionary constraints. Behavioral Ecology 24:806-811.

Doebeli, M., and U. Dieckmann. 2003. Speciation along environmental gradients. Nature 421:259-264.

Doebeli, M., and I. Ispolatov. 2010. Complexity and diversity. Science 328:494- 497.

Eklöv, P., and R. Svanbäck. 2006. Predation risk influences adaptive morphological variation in fish populations. American Naturalist 167:440-452.

Evans, S. N., P. L. Ralph, S. J. Schreiber, and A. Sen. 2013. Stochastic population growth in spatially heterogeneous environments. Journal of Mathematical Biology 66:423-476.

Felsenstein, J. 1988. Phylogenies and quantitative characters. Annual Review of Ecology and Systematics 19:445-471.

Gilmour, A. R., B. J. Gogel, B. R. Cullis, and R. Thompson. 2009. ASReml user guide release 3.0. VSN International Ltd, Hemel Hempstead, UK.

Hadfield, J. D. 2010. MCMC methods for multi-response generalized linear mixed models: the MCMCglmm R package. Journal of Statistical Software 33:1-22.

Hammerschlag-Peyer, C. M., and C. A. Layman. 2010. Intrapopulation variation in habitat use by two abundant coastal fish species. Marine Ecology Progress Series 415:211-220.

Hansen, T. F., and D. Houle. 2008. Measuring and comparing evolvability and constraint in multivariate characters. Journal of Evolutionary Biology 21:1201-1219.
Harrod, C., J. Mallela, and K. K. Kahilainen. 2010. Phenotypeenvironment correlations in a putative whitefish adaptive radiation. Journal of Animal Ecology 79:1057-1068.

Hart, S. P., S. J. Schreiber, and J. M. Levine. 2016. How variation between individuals affects species coexistence. Ecology Letters 19:825-838.

Hatase, H., K. Omuta, and K. Tsukamoto. 2007. Bottom or midwater: alternative foraging behaviours in adult female loggerhead sea turtles. Journal of Zoology 273:46-55.

Hatase, H., K. Omuta, and K. Tsukamoto. 2010. Oceanic residents, neritic migrants: a possible mechanism underlying foraging dichotomy in adult female loggerhead turtles (Caretta caretta). Marine Biology 157:1337-1342.

Hawley, K. L., C. M. Rosten, G. Christensen, and M. C. Lucas. 2016. Fine-scale behavioural differences distinguish resource use by ecomorphs in a closed ecosystem. Scientific Reports 6:24369.

Hughes, A., B. Inouye, M. Johnson, N. Underwood, and M. Vellend. 2008. Ecological consequences of genetic diversity. Ecology Letters 11:609-623.

Hutchinson, G. E. 1957. Concluding remarks. Cold Spring Harbor Symposia on Quantitative Biology 22:415-427.

Ingram, T., R. Svanbäck, N. J. B. Kraft, P. Kratina, L. Southcott, and D. Schluter. 2012. Intraguild predation drives evolutionary niche shift in threespine stickleback. Evolution 66:1819-1832.

Jackson, A. L., R. Inger, A. C. Parnell, and S. Bearhop. 2011. Comparing isotopic niche widths among and within communities: SIBER - Stable Isotope Bayesian Ellipses in R. Journal of Animal Ecology 80:595-602.

Jensen, J. 1906. Sur les fonctions convexes et les inégalités entre les valeurs moyennes. Acta Mathematica 30:175-193.

Johnson, P. C. D., S. J. E. Barry, H. M. Ferguson, and P. Muller. 2015. Power analysis for generalized linear mixed models in ecology and evolution. Methods in Ecology and Evolution $6: 133-142$.

Jones, A. W., and D. M. Post. 2016. Does intraspecific competition promote variation? A test via synthesis. Ecology and Evolution 6:1646-1655

Kernaléguen, L., B. Cazelles, J. P. Y. Arnould, P. Richard, C. Guinet, and Y. Cherel. 2012. Long-term species, sexual and individual variations in foraging strategies of fur seals revealed by stable isotopes in whiskers. PLoS ONE 7: e32916.

Kernaléguen, L., J. P. Y. Arnould, C. Guinet, and Y. Cherel. 2015. Determinants of individual foraging specialization in large marine vertebrates, the Antarctic and subantarctic fur seals. Journal of Animal Ecology 84:1081-1091.

Kingsolver, J. G., H. E. Hoekstra, J. M. Hoekstra, D. Berrigan, S. N. Vignieri, C. E. Hill, A. Hoang, P. Gibert, and P. Beerli. 2001. The strength of phenotypic selection in natural populations. American Naturalist 157:245-261.

Knudsen, R., R. Primicerio, P.-A. Amundsen, and A. Klemetsen. 2010. Temporal stability of individual feeding specialization may promote speciation. Journal of Animal Ecology 79: $161-168$.

Kobler, A., G. E. Maes, Y. Humblet, F. A. Volckaert, and M. Eens. 2011. Temperament traits and microhabitat use in bullhead, Cottus perifretum: fish associated with complex habitats are less aggressive. Behaviour 148:603-625.

Lande, R., and S. Arnold. 1983. The measurement of selection on correlated characters. Evolution 37:1210-1226.

Layman, C. A., et al. 2012. Applying stable isotopes to examine food-web structure: an overview of analytical tools. Biological Reviews 87:545-562. 
Letten, A. D., P. J. Ke, and T. Fukami. 2016. Linking modern coexistence theory and contemporary niche theory. Ecological Monographs 87:161-177.

Lowther, A. D., and S. D. Goldsworthy. 2011. Detecting alternate foraging ecotypes in Australian sea lion (Neophoca cinerea) colonies using stable isotope analysis. Marine Mammal Science 27:567-586.

MacArthur, R., and R. Levins. 1967. The limiting similarity, convergence, and divergence of coexisting species. American Naturalist 101:377-385.

Madansky, A. 1959. Bounds on the expectation of a convex function of a multivariate random variable. Annals of Mathematical Statistics 30:743-746.

Maldonado, K., F. Bozinovic, S. D. Newsome, and P. Sabat. 2017. Testing the niche variation hypothesis in a community of passerine birds. Ecology 98:903-908.

Martin, T. L., and R. B. Huey. 2008. Why "suboptimal" is optimal: Jensen's inequality and ectotherm thermal preferences. American Naturalist 171:E102-E118.

Martin, C., I. Bentaleb, S. Steelandt, and C. Guinet. 2011. Stable carbon and nitrogen isotope variations in canine dentine growth layers of Kerguelen southern elephant seals. Marine Ecology Progress Series 439:295-305.

Masello, J. F., M. Wikelski, C. C. Voigt, and P. Quillfeldt. 2013. Distribution patterns predict individual specialization in the diet of dolphin gulls. PLoS ONE 8:e67714.

Matich, P., M. R. Heithaus, and C. A. Layman. 2011. Contrasting patterns of individual specialization and trophic coupling in two marine apex predators. Journal of Animal Ecology 20:294-305.

Matthews, B., K. Marchinko, D. Bolnick, and A. Mazumder. 2010. Specialization of trophic position and habitat use by sticklebacks in an adaptive radiation. Ecology 91:1025-1034.

McCann, K. S., J. B. Rasmussen, and J. Umbanhowar. 2005. The dynamics of spatially coupled food webs. Ecology Letters 8:513-523.

McGuigan, K., S. F. Chenoweth, and M. W. Blows. 2005. Phenotypic divergence along lines of genetic variance. American Naturalist 165:32-43.

McIntyre, T., C. A. Tosh, J. Plötz, H. Bornemann, and M. N. Bester. 2010. Segregation in a sexually dimorphic mammal: a mixed-effects modelling analysis of diving behaviour in southern elephant seals. Marine Ecology Progress Series 412:293-304.

Miller, S. E., D. Metcalf, and D. Schluter. 2015. Intraguild predation leads to genetically based character shifts in the threespine stickleback. Evolution 69:3194-3203.

Moran, E. V., F. Hartig, and D. M. Bell. 2016. Intraspecific trait variation across scales: implications for understanding global change responses. Global Change Biology 22:137-150.

Mousseau, T. A., and D. A. Roff. 1987. Natural selection and the heritability of fitness components. Heredity 59:181-197.

Nakagawa, S., and H. Schielzeth. 2010. Repeatability for Gaussian and non-Gaussian data: a practical guide for biologists. Biological Reviews 85:935-956.

Newsome, S. D., M. T. Tinker, D. H. Monson, O. T. Oftedal, K. Ralls, M. M. Staedler, M. L. Fogel, and J. A. Estes. 2009. Using stable isotopes to investigate individual diet specialization in California sea otters (Enhydra lutris nereis). Ecology 90:961-974.

Newsome, S. D., M. T. Tinker, V. A. Gill, Z. N. Hoyt, A. Doroff, L. Nichol, and J. L. Bodkin. 2015. The interaction of intraspecific competition and habitat on individual diet specialization: a near range-wide examination of sea otters. Oecologia 178:45-59.

Nosil, P. 2012. Ecological speciation. Oxford University Press, Oxford, UK.
Nosil, P., and C. P. Sandoval. 2008. Ecological niche dimensionality and the evolutionary diversification of stick insects. PLoS ONE 3:e1907.

Nosil, P., L. J. Harmon, and O. Seehausen. 2009. Ecological explanations for (incomplete) speciation. Trends in Ecology \& Evolution 24:145-156.

Novak, M., and M. T. Tinker. 2015. Timescales alter the inferred strength and temporal consistency of intraspecific diet specialization. Oecologia 178:61-74.

Peckham, S. H., D. Maldonado-Diaz, Y. Tremblay, R. Ochoa, J. Polovina, G. Balazs, P. H. Dutton, and W. Nichols. 2011. Demographic implications of alternative foraging strategies in juvenile loggerhead turtles Caretta caretta of the North Pacific Ocean. Marine Ecology Progress Series 425:269-280.

Phillips, P. C., and S. J. Arnold. 1999. Hierarchical comparison of genetic variance-covariance matrices. I. Using the Flury hierarchy. Evolution 53:1506-1515.

Pianka, E. R. 1973. The structure of lizard communities. Annual Review of Ecology and Systematics 4:53-74.

Pimm, S. L., S. Alibhai, R. Bergl, A. Dehgan, C. Giri, Z. Jewell, L. Joppa, R. Kays, and S. Loarie. 2015. Emerging technologies to conserve biodiversity. Trends in Ecology \& Evolution 30:685-696.

Potier, S., A. Carpentier, D. Grémillet, B. Leroy, and A. Lescroël. 2015. Individual repeatability of foraging behaviour in a marine predator, the great cormorant, Phalacrocorax carbo. Animal Behaviour 103:83-90.

Quevedo, M., R. Svanbäck, and P. Eklöv. 2009. Intrapopulation niche partitioning in a generalist predator limits food web connectivity. Ecology 90:2263-2274.

R Core Team. 2014. R: a language and environment for statistical computing. R Foundation for Statistical Computing, Vienna, Austria.

Ramírez, I., V. H. Paiva, I. Fagundes, D. Menezes, I. Silva, F. R. Ceia, R. A. Phillips, J. A. Ramos, and S. Garthe. 2016. Conservation implications of consistent foraging and trophic ecology in a rare petrel species. Animal Conservation 19: 139-152.

Rice, W. R., and E. E. Hostert. 1993. Laboratory experiments on speciation: what have we learned in 40 years? Evolution 47:1637-1653.

Robinson, G. E., and R. E. Jr Page. 1989. Genetic determination of nectar foraging, pollen foraging, and nest-site scouting in honey bee colonies. Behavioral Ecology and Sociobiology 24:317-323.

Rolshausen, G., G. Segelbacher, C. Hermes, K. A. Hobson, and H. M. Schaefer. 2013. Individual differences in migratory behavior shape population genetic structure and microhabitat choice in sympatric blackcaps (Sylvia atricapilla). Ecology and Evolution 3:4278-4289.

Rooney, N., K. McCann, G. Gellner, and J. C. Moore. 2006. Structural asymmetry and the stability of diverse food webs. Nature 442:265-268.

Rosenblatt, A. E., and M. R. Heithaus. 2011. Does variation in movement tactics and trophic interactions among American alligators create habitat linkages? Journal of Animal Ecology 80:786-798.

Rossman, S., P. H. Ostrom, M. Stolen, N. B. Barros, H. Gandhi, C. A. Stricker, and R. S. Wells. 2015. Individual specialization in the foraging habits of female bottlenose dolphins living in a trophically diverse and habitat rich estuary. Oecologia 178:415-425.

Roughgarden, J. 1972. Evolution of niche width. American Naturalist 106:683-718.

Roughgarden, J. 1974. Niche width: biogeographic patterns among Anolis lizard populations. American Naturalist 108:429-442. 
Roughgarden, J. 1979. Theory of population genetics and evolutionary ecology: an introduction. Macmillan, New York, New York, USA.

Ruel, J., and M. Ayres. 1999. Jensen's inequality predicts effects of environmental variation. Trends in Ecology \& Evolution 14:361-366.

Schluter, D. 1996. Adaptive radiation along genetic lines of least resistance. Evolution 50:1766-1774.

Schluter, D. 2009. Evidence for ecological speciation and its alternative. Science 323:737-741

Smith, B. R., and D. T. Blumstein. 2008. Fitness consequences of personality: a meta-analysis. Behavioral Ecology 19: $448-455$.

Snowberg, L. K., K. M. Hendrix, and D. I. Bolnick. 2015. Covarying variances: more morphologically variable populations also exhibit more diet variation. Oecologia 178:89-101.

Steppan, S. J., P. C. Phillips, and D. Houle. 2002. Comparative quantitative genetics: evolution of the $G$ matrix. Trends in Ecology \& Evolution 17:320-327.

Stuart, Y. E., T. S. Campbell, P. A. Hohenlohe, R. G. Reynolds, L. J. Revell, and J. B. Losos. 2014. Rapid evolution of a native species following invasion by a congener. Science 346 : 463-466.

Svanbäck, R., and D. I. Bolnick. 2007. Intraspecific competition drives increased resource use diversity within a natural population. Proceedings of the Royal Society B: Biological Sciences 274:839-844.

Svanbäck, R., M. Quevedo, J. Olsson, and P. Eklöv. 2015. Individuals in food webs: the relationships between trophic position, omnivory and among-individual diet variation. Oecologia 178:103-114.

Swanson, H. K., M. Lysy, M. Power, A. D. Stasko, J. D Johnson, and J. D. Reist. 2015. A new probabilistic method for quantifying n-dimensional ecological niches and niche overlap. Ecology 96:318-324.

Tilman, D. 1982. Resource competition and community structure. Princeton University Press, Princeton, New Jersey, USA.
Tinker, M. T., P. R. Guimarães, M. Novak, F. M. D. Marquitti, J. L. Bodkin, M. Staedler, G. Bentall, and J. A. Estes. 2012. Structure and mechanism of diet specialisation: testing models of individual variation in resource use with sea otters. Ecology Letters 15:475-483.

Vander Zanden, M. J., J. M. Casselman, and J. B. Rasmussen. 1999. Stable isotope evidence for the food web consequences of species invasions in lakes. Nature 401:464 467.

Violle, C., B. J. Enquist, B. J. McGill, L. Jiang, C. H. Albert, C. Hulshof, V. Jung, and J. Messier. 2012. The return of the variance: intraspecific variability in community ecology. Trends in Ecology \& Evolution 27:244-252.

Voslamber, B., M. Platteeuw, and M. R. van Eerden. 2010. Individual differences in feeding habits in a newly established Great Egret Casmerodius albus population: key factors for recolonisation. Ardea 98:355-363.

Wakefield, E. D., I. R. Cleasby, S. Bearhop, T. W. Bodey, R. D. Davies, P. I. Miller, J. Newton, S. C. Votier, and K. C. Hamer. 2015. Long-term individual foraging site fidelity-why some gannets don't change their spots. Ecology 96:3058-3074.

Whitham, T. G., et al. 2006. A framework for community and ecosystem genetics: from genes to ecosystems. Nature Reviews Genetics 7:510-523.

Whittaker, R. H., S. A. Levin, and R. B. Root. 1973. Niche, habitat, and ecotope. American Naturalist 107:321-338.

Wilson, D. S., K. Coleman, and A. B. Clark. 1993. Shy-bold continuum in pumpkinseed sunfish (Lepomis gibbosus): an ecological study of a psychological trait. Journal of Comparative Psychology 107:250-260.

Woo, K. J., K. H. Elliott, M. Davidson, A. J. Gaston, and G. K. Davoren. 2008. Individual specialization in diet by a generalist marine predator reflects specialization in foraging behaviour. Journal of Animal Ecology 77:1082-1091.

Zandona, E., S. K. Auer, S. S. Kilham, J. L. Howard, A. LopezSepulcre, M. P. O'Connor, R. D. Bassar, A. Osorio, C. M. Pringle, and D. N. Reznick. 2011. Diet quality and prey selectivity correlate with life histories and predation regime in Trinidadian guppies. Functional Ecology 25:964-973.

\section{SUPPORTING INFORMATION}

Additional supporting information may be found in the online version of this article at http://onlinelibrary.wiley.com/doi/ 10.1002/ecy.2129/suppinfo 